\section{The Research Support Facility Data Center: An Example of Best Practices Implementation}

\author{
The National Renewable Energy Laboratory \\ (NREL) is world-renowned for its commitment \\ to green building construction. To further this \\ commitment to green building and leading by \\ example, NREL included an ultra-energy-efficient \\ data center in the laboratory's new Research \\ Support Facility (RSF), which recently received a \\ Leadership in Energy and Environmental Design ${ }^{\circledR}$ \\ (LEED) Platinum designation from the U.S. Green \\ Building Council.
}

\section{Design}

To support the RSF's net zero energy goals, NREL's data center was designed with a strategy to minimize energy footprint, without compromising service quality for NREL staff. NREL's Information Technology (IT) and building technologies experts were engaged throughout the design process to integrate data center needs into the design of the RSF building. The approach relied on a whole systems design to ensure the data center would have a symbiotic relationship with building mechanical systems and operations, including staff interaction with IT systems. Even with the addition of the campus-wide data center, the RSF's energy use is $50 \%$ less than current commercial building code.

Planners recognized that from an energy efficiency standpoint, data centers are facing a power and cooling crisis. They use $2-3 \%$ of all power produced in the United States, and power demands are increasing. NREL's data center was designed to optimize efficiency by implementing building and data center best practices to support the RSF's energy goals and serve as an example of how data centers can operate sustainably. Figure 1 shows an example of just one of the energy efficiency best practices implemented in the data center-ENERGY STAR ${ }^{\circledR}$-rated blade servers-rather than the use of energy-intensive stand-alone servers. Blade servers, coupled with server virtualization, have lessened energy consumption by approximately $90 \%$ to support the same workload of NREL's legacy data systems.

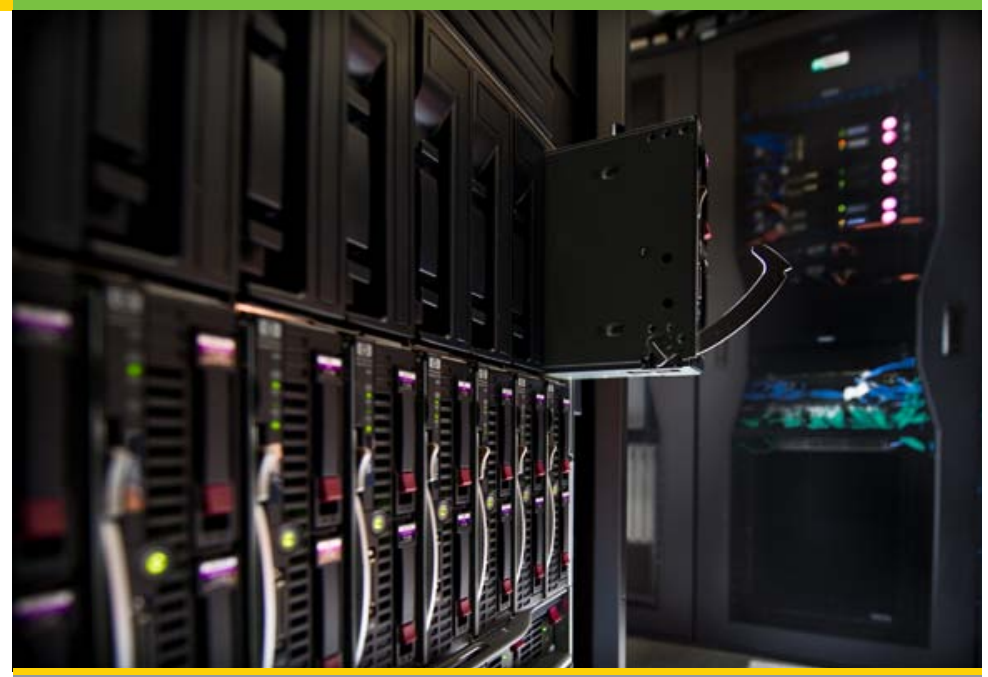

Figure 1. An example of RSF data center best practice implementation. Photo by Dennis Schroeder, NREL/PIX 18765

\section{Energy and Cost Reductions}

Over the past two years, NREL has reduced per-user data center energy requirements by $81 \%$, resulting in an annual cost savings of $\$ 200,000$ in utility bills (at $\$ 0.06$ per kilowatt hours), and an annual reduction in carbon dioxide emissions of nearly 5,000,000 pounds. NREL achieved this result by implementing the industry's most energy-efficient data center best practices into an large administrative office building designed to show that the interconnectivity of design, planning, and staff support can realize the impossible.

Industry and other governmental organizations have taken notice of NREL's data center accomplishments and innovation, supporting NREL's leadership role in green data center design and sustainable IT operations.

\section{Power Usage Effectiveness}

Power Usage Effectiveness (PUE) is the industry standard metric used to measure the energy efficiency of data centers, including the combined power usage from cooling, power systems, and equipment. PUE is calculated as a ratio using the formula:

\section{PUE $=($ Cooling + Power + Equipment $) /$ Equipment}

The PUE for NREL's legacy data center was estimated to be 3.3. In contrast, the measured PUE for NREL's RSF data center ranges from 1.11-1.16 during the cold winter months where chilled water cooling is not required. The PUE rises slightly to $1.20-1.25$ in the warmer months. The extremely low PUE ranks among the lowest found in data centers worldwide. Figure 2 illustrates that data center administrators are constantly analyzing and optimizing the cooling systems to improve PUE. 


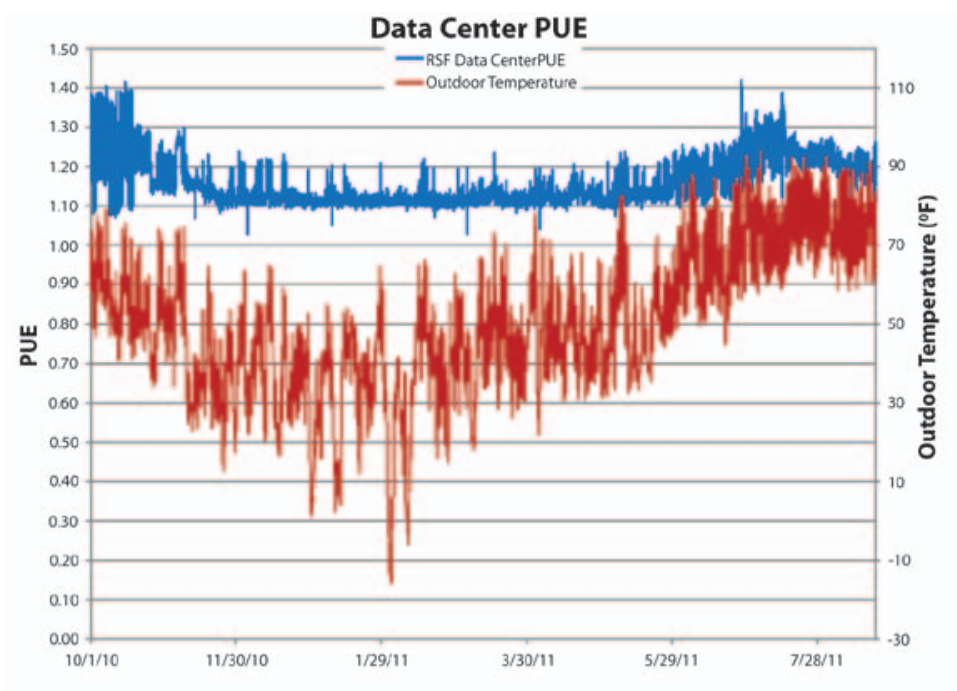

Figure 2. RSF data center PUE. Average PUE is 1.16

Figure 3 contrasts the energy requirements for cooling, power systems, and equipment between the RSF data center and NREL's legacy data center. Figure 4 shows a comparison of the PUE from the legacy data center with the RSF data center. These graphs illustrate that energy requirements for cooling and power systems have been heavily optimized with the best practices implemented in the RSF data center.

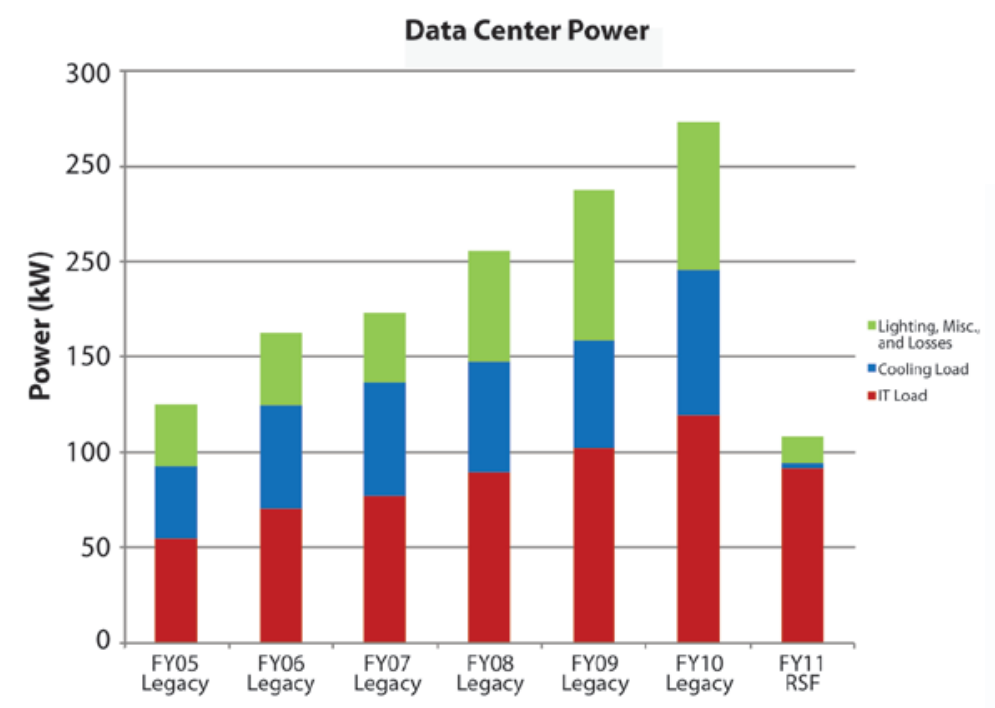

Figure 3. Energy requirements for cooling

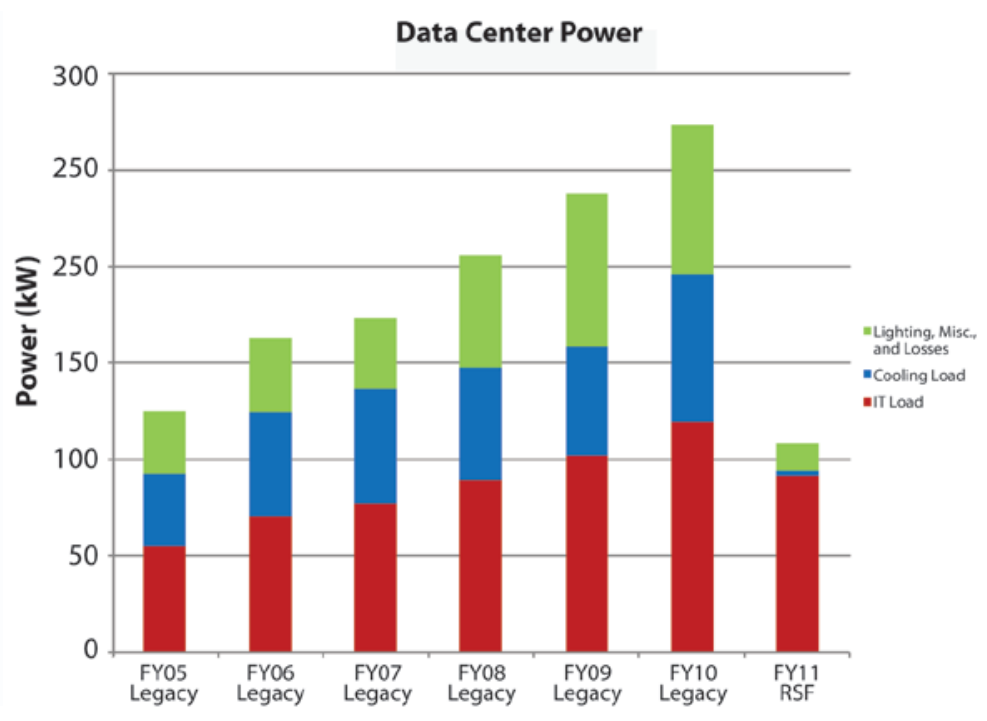

Figure 4. PUE comparison of RSF data center to NREL's legacy data center

\section{Beyond PUE}

PUE measures how well an organization has optimized its energy use for data center cooling and power systems. It does not take into account efforts to optimize energy use for servers, storage, and network infrastructure running within the data center. Comparing watts per user for total data center power consumption provides a more comprehensive evaluation of overall data center energy efficiency. Figure 5 compares watts per user for the legacy data center with the RSF data center.

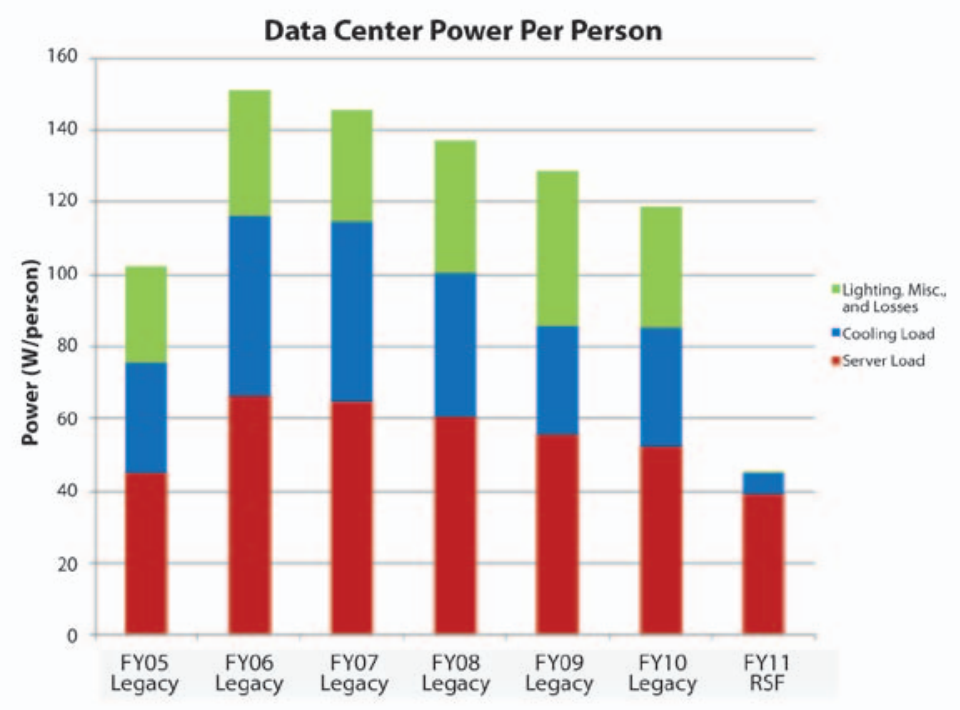

Figure 5. RSF data center's watts per user compared to the legacy data center 


\section{Fast Facts}

Data center opened: July 2010

Industry standard PUE: 2.0-3.0

Data center expected PUE: 1.50

RSF Data center PUE: 1.1-1.16 (continually tuning)

Power usage decrease to baseline: $81 \%$

(as a function of users)

Annual savings: $\$ 200,000$ (at $\$ 0.06 /$ kilowatt hours)

Annual $\mathbf{C O}_{2}$ savings: $5,000,000$ pounds

\section{Air Handling and Temperature Management}

In typical data centers you can feel the energy consumption from racks of servers radiating heat, while icy air blows through the room to cool them. The data center's fully contained hot aisle configuration minimizes this problem. The configuration includes effective air-side economizer cooling with an evaporative boost when needed. Waste heat is also captured for use throughout the RSF building during the colder months.

\section{Free Cooling}

NREL is located in a climate that is favorable for "free cooling" - using outside air to provide the majority of the data center's cooling needs. The cooling system for the RSF data center was designed to minimize the use of traditional air conditioners. Based on this historical climate data, NREL's cooling system was designed to use direct air and evaporative cooling methods for almost the entire year. The system was designed to provide cooling to the data center ranging from $65^{\circ} \mathrm{F}-80^{\circ} \mathrm{F}$, with humidity ranging from $20-60 \%$. Free cooling is available for a significant number of days a year (except for the most hot and humid days) without air conditioning.

\section{Waste Heat Reuse}

The equipment racks for the data center are designed with hot aisle containment. Air from the hot aisle is extracted from the data center for reuse in heating the RSF building in colder months, when required. The heat from the data center is either ducted to the building's ventilation systems, or exhausted to the outside when not needed. Figure 6 shows the hot aisle containment system used in NREL's RSF data center. Supply air is not mixed with hot air, minimizing the need for active cooling methods. Figure 7 illustrates the data center air handling configuration.

\section{Uninterruptible Power Supply}

The data center uses a $97 \%$ efficient $500 \mathrm{~kW}$ uninterruptable power supply (UPS) to supply 15 minutes of backup power to servers, storage, and network gear located in the RSF data center. The UPS was designed to perform two functions: 1) condition line power, and 2) sustain the data center until the emergency power generator comes on.

The UPS is configured with $125 \mathrm{~kW}$ worth of batteries and scales in $25 \mathrm{~kW}$ increments. Ultra-efficient power distribution units (PDU's) are used to distribute power to the equipment racks within the data center. In contrast, the UPS used in NREL's legacy data center was only $80 \%$ efficient and produced excess heat, which required additional cooling. The new UPS is effectively saving about $37 \mathrm{~kW}$ of energy, while running a $100 \mathrm{~kW}$ equipment load.

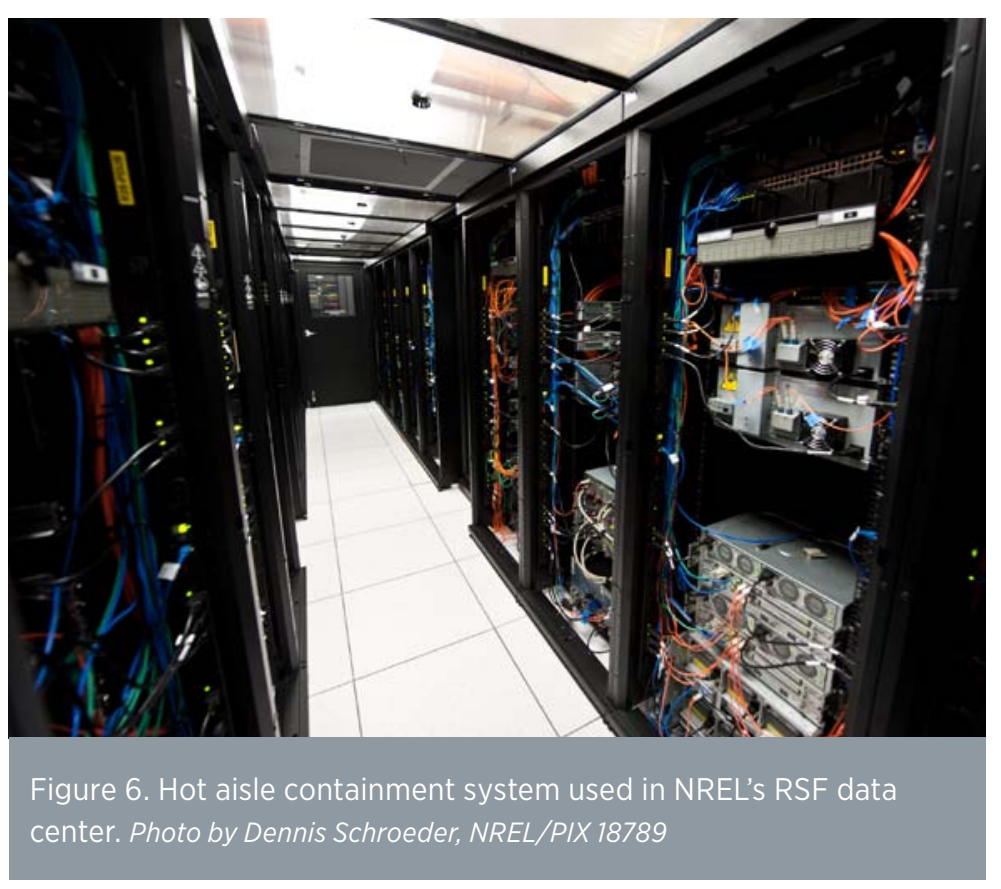




\section{Equipment Environment}

The RSF reduced energy consumption by approximately $65 \%$ through the implementation of blade servers and server virtualization to support the same workload of the legacy systems. Plug loads in the RSF were also minimized with extensive use of laptops and high-efficiency office equipment.

\section{Server Environment and Virtualization}

To support the energy goals for the RSF, data center planners avoided over provisioning capacity for IT equipment. They also determined to implement newer technologies to save energy.

NREL replaced $90 \%$ of its legacy server environment with HP Blade servers that utilize variable speed fans and energyefficient power supplies. Additionally, NREL implemented Vmware ${ }^{\circledR}$, a virtualized server environment application, to decrease the number of physical servers needed for operations.
Currently, 70\% of NREL's server environment is virtualized. The original goal was to reach a 20:1 ratio for server virtualization, meaning that the workload that used to run on 20 physical servers would only require one single blade server. At this ratio, the energy footprint is reduced by more than $96 \%$ for each server. However, in some environments, NREL has experienced as much as a 29:1 ratio. Storage area networks (SAN's) are used to pool storage resources in an effort to reduce the amount of hardware that would typically be required for storage dedicated to server resources.

\section{Metering}

Metering was installed to continually monitor data center performance and provide for real-time optimization of energy usage. Performance measures are reviewed regularly and opportunities for improvement are identified.

\section{For More Information}

More detailed information about the RSF data center can be found on NREL's Web site www.nrel.gov/rsf, or by contacting NREL's Public Affairs Office: 303-275-4090.

Figure 7. RSF data center cooling and reuse of heat from hot aisle containment system

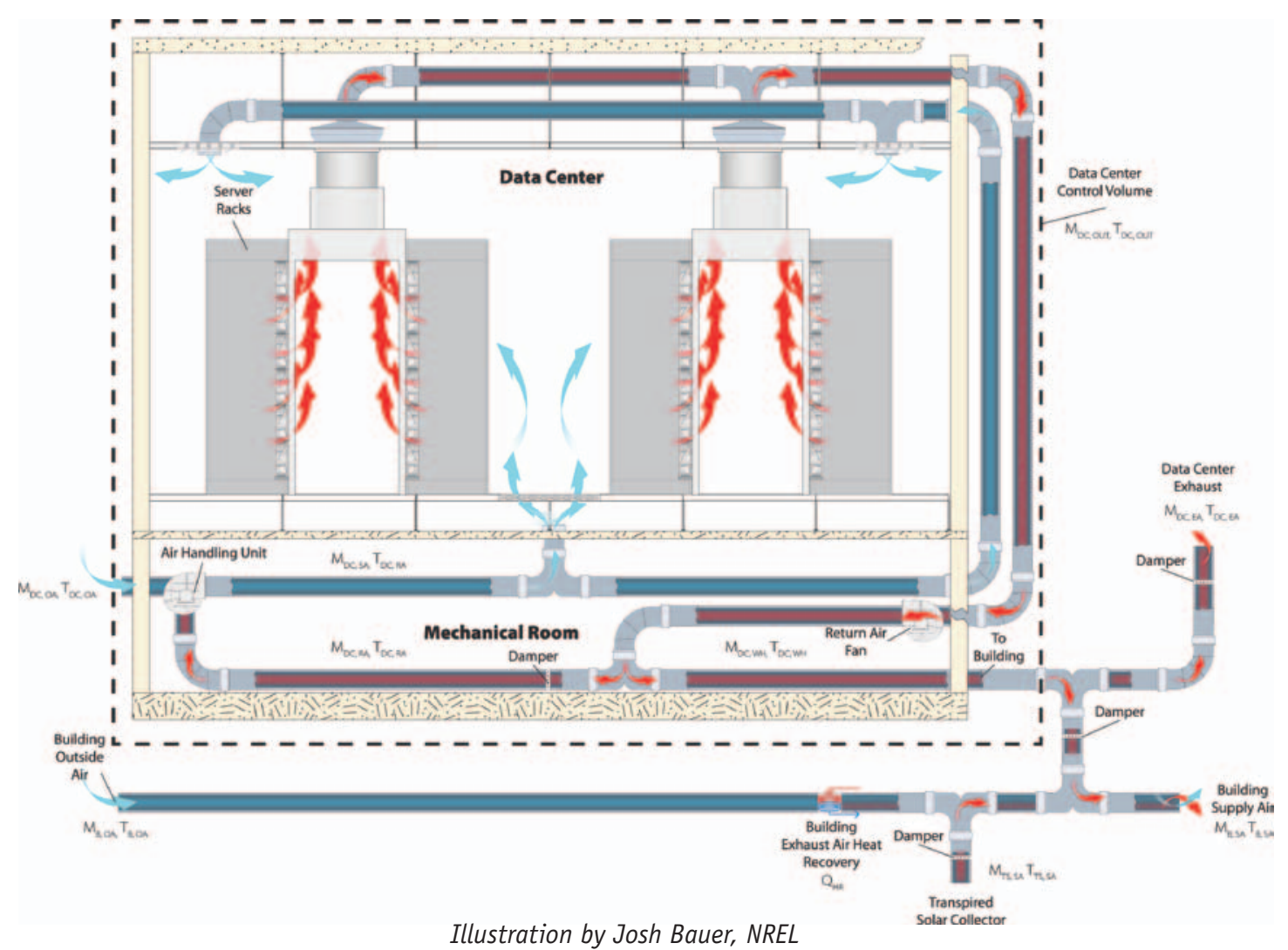

(ascheres ENERGY

Energy Efficiency \& Renewable Energy
EERE Information Center

1-877-EERE-INFO (1-877-337-3463)

www.eere.energy.gov/informationcenter

Printed with a renewable-source ink on paper containing at least $50 \%$ wastepaper, including $10 \%$ post consumer waste.
Prepared by the National Renewable Energy Laboratory (NREL), a national laboratory of the U.S. Department of Energy, Office of Energy Efficiency and Renewable Energy; NREL is operated by the Alliance for Sustainable Energy, LLC. 